\section{Von hilflosen Bauern und mutigen Aktivistinnen}

\section{Kirchliche Entwicklungszusammenarbeit postkolonial be-} leuchtet

Isabelle Knobel

Durch die «Black Lives Matter»-Bewegung nahmen 2020 die öffentlichen Diskussionen zu Rassismus auch in der Schweiz stark zu. Auf unterschiedlichen Ebenen wurden die Debatten geführt, von Schokoküssen bis hin zu der Frage nach strukturellem Rassismus. Ich schrieb meine Masterarbeit mit dem Titel «Von hilflosen Bauern und mutigen Aktivistinnen Kirchliche Entwicklungszusammenarbeit postkolonial beleuchtet» ${ }^{1}$ vor den Geschehnissen, die durch den Tod George Floyds ausgelöst wurden. In diesem Beitrag stelle ich die Arbeit und deren Haupterkenntnisse vor. Dabei wird sich zeigen, dass die bearbeitete Problemstellung viel mit der Frage nach strukturellem Rassismus zu tun hat.

\section{Problemfeld und Fragestellung}

Wie viele andere humanitäre oder soziale Bewegungen hat die Hilfsindustrie versagt, antirassistische Arbeit in ihre Strategie aufzunehmen. Das ist problematisch. Auf der einen Seite richtet sie ihren Fokus auf die Ungleichheit zwischen Nord und Süd, auf die Ungerechtigkeit zwischen denen, die kolonisiert haben und denen, die kolonialisiert wurden. Gleichzeitig basieren ihre Kampagnen auf der Förderung von kolonialen Fan-

1 Isabelle Knobel, Von hilflosen Bauern und mutigen Aktivistinnen. Kirchliche Entwicklungszusammenarbeit postkolonial beleuchtet, Bern 2020.

Jahrbuch Diakonie Schweiz 4 (2020/2021) - ISSN 2504-3994

Dieser Text ist lizenziert unter einer Creative Commons Namensnennung 4.0 International Lizenz (CC BY 4.0): (https://creativecommons.org/licenses/by/4.0/)

tasien und deshalb auch auf dem Aufrechterhalten von Rassismus. Das ist ein Widerspruch: Ungleichheit zu thematisieren, aber zur gleichen Zeit durch ihre Arbeit Ungleichheit hervorzurufen. ${ }^{2}$

Die portugiesische Theoretikerin Grada Kilomba beschreibt mit diesem Zitat treffend, mit welchem Problemfeld sich die Masterarbeit beschäftigt. Kirchliche Entwicklungszusammenarbeit (EZA) muss, will sie glaubwürdig bleiben, diese Problematik unbedingt ernst nehmen und ihr Engagement aus postkolonialer und rassismuskritischer Sicht stetig weiterentwickeln: «Ansonsten läuft sie Gefahr, ihrer eigenen Arbeit entgegenzuwirken oder, wie Kilomba es eben ausdrückt, «Ungleichheit zu thematisieren, aber zur gleichen Zeit (...) Ungleichheit hervorzurufen.) Dies gilt nicht nur für die Kampagnenarbeit der EZA-Organisationen, sondern auch für die gesamte Projektarbeit, die Struktur, die Kommunikation und die Zusammenarbeit mit den Partnerorganisationen im globalen Süden.» ${ }^{3}$

Die postkolonialen Theorien wurden bereits an verschiedenen Stellen für die Theologie fruchtbar gemacht. Im Gegensatz zu ihrem Stellenwert in anderen Fachgebieten bleiben sie in der Theologie ein Randthema. Die diesem Beitrag zugrunde liegende Masterarbeit zeigt auf, dass postkoloniale Theorien gewinnbringend mit kirchlicher EZA verbunden werden können. Die Leitfrage lautet:

Wie lässt sich kirchliche EZA mit postkolonialen Theorien verbinden und welche Chancen bietet dies? Wo zeigen sich allenfalls Grenzen?

In einem ersten Schritt wurden die zur Beantwortung der Fragen nötigen, theoretischen Grundlagen erarbeitet. In diesem Artikel wird kurz auf diese

2 Grada Kilomba, in: Carolin Philipp / Timo Kiesel, White charity. Schwarzsein und Weisssein auf Spendenplakaten, 2012. URL: www.whitecharity.de (Übersetzung IK, abgerufen am 13.03.2021).

3 Knobel, Von hilflosen Bauern (Anm. 1), 5.

Jahrbuch Diakonie Schweiz 4 (2020/2021) http://dx.doi.org/10.22018/JDS.2021.15 
Grundlagen geschaut, um dann ausführlicher auf das eigentliche Kernstück der Arbeit einzugehen: die Analyse dreier HEKS-Kampagnen.

\section{Theoretische Grundlagen}

Ökumenische Diakonie

Das erste Kapitel beschäftigt sich mit der ökumenischen Diakonie und ihrer Geschichte. Dabei liegt der Fokus auf der EZA und ihrem Wandel innerhalb der ökumenischen Diakonie. Das Kapitel zeigt auf, dass EZA ein wichtiger Teil der ökumenischen Diakonie bildet. Mit der ACT Alliance wird zudem ein konkretes, aktuelles Beispiel einer ökumenischen, entwicklungspolitischen Organisation kurz angeschaut - bereits mit einer postkolonialen Perspektive im Hintergrund.

Das Kapitel zeigt auf, wie sich die ökumenische Diakonie stetig weiterentwickelt hat. Wichtig erscheint mir dabei besonders die Fokusverschiebung von der Wohltätigkeit hin zu Gerechtigkeit. Darin zeigt sich auch eine zunehmende postkoloniale Sensibilität und damit eine Unterstützung, die die Machtverhältnisse immer mehr einzuebnen versucht.

\section{Protestantische EZA in der Schweiz.}

Von dem grossen Kontext der ökumenischen Diakonie hin zu einem konkreten Kontext, der mit dieser verbunden ist: Die Protestantische EZA in der Schweiz. Diese stellt zugleich den Rahmen, in dem sich der Analysegegenstand befindet.

Hier hat sich vor allem gezeigt, dass protestantische EZA mit vielen politischen und ökonomischen Schwierigkeiten zu kämpfen hat. Die Fusion von HEKS und Bfa lässt sich als Versuch lesen, sich als kirchliche EZA in diesem Kontext zu behaupten.

\section{Postkoloniale Theorien}

Die theoretische Brille der Analyse bieten die postkolonialen Theorien. Diese machen Nachwirkungen des Kolonialismus und immer noch oder neu existierende kritische Machtverhältnisse sichtbar. Ein Schwerpunkt liegt dabei auf kulturellen Aspekten. Grundlegend für die postkolonialen Theorien ist die Annahme, dass stereotype, kolonialistische Denkmuster noch heute nachwirken. Was eine solche postkoloniale Analyse-Perspektive genau bedeutet, wird in den Ausführungen zur Kampagnen-Analyse deutlich werden.

Im Kapitel zu den postkolonialen Theorien zeigt sich zudem, dass auch die Schweiz sich mit postkolonialer Kritik auseinandersetzen muss: Das hier abgedruckte Werbeplakat von Lindt aus dem Jahr 1933 (Abb. 1) spricht für sich: «Rassig, braun und süss und fein, wie dies kleine Negerleiny.

4 URL: https://www.nzz.ch/feuilleton/schweiz-und-kolonialismus-so-weiss-war-dasland-nicht-ld.1570208? reduced=true (abgerufen am 13.03.2021).
Jahrbuch Diakonie Schweiz $4(2020 / 2021)$ http://dx.doi.org/10.22018/JDS.2021.15 


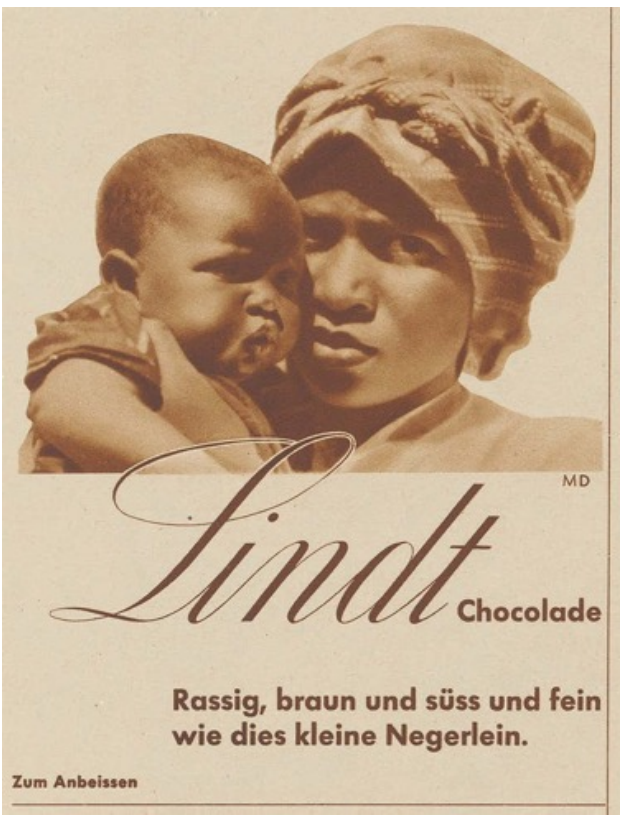

Abb. 1: Koloniale Schweiz

\section{Kampagnen-Analyse HEKS}

Kampagnen und Methodik.

Bewusst wurden drei sehr unterschiedliche HEKS-Kampagnen gewählt. Bei der ersten handelt es sich um die bereits seit 2007 laufende, öffentlichkeitswirksame Kampagne «Hilfe schenken». Symbolisch wird hier eine Urkunde geschenkt, während die Menschen in den HEKS-Projekten weltweit das eigentliche Geschenk erhalten. Die beiden anderen Kampagnen gehörten zu den jährlich wechselnden Sammelkampagnen: «Gloria Frauen für den Frieden in Kolumbien» war die Kampagne des Jahres 2018, «Korenkombu - Hoffnung für die Urbevölkerung Indiens» war im Jahr 2019 dran. Letztere porträtierte die Adivasi, ihre Lebensweise im Einklang mit der Natur und ihr Kampf um Landrechte. Bei «Gloria» standen die kolumbianische «Organización Femenina Popular» und die Menschenrechtsaktivistin Gloria Suárez im Fokus. Die Kampagne zeigte deren gefährliches Engagement für Frauen und den Frieden in ihrem Land.

Das methodische Grundraster der Analyse wurde aus einem Artikel ${ }^{5}$ von Carolin Philipp heraus entwickelt. Für einen Dokumentarfilm analysierte sie zusammen mit Timo Kiesel entwicklungspolitische Spendenwerbung in Deutschland aus postkolonialer und rassismuskritischer Perspektive. Die Auswertung führte sie zu sieben kritischen, inhaltlichen Gegensätzen in der Konstruktion des «Wir» und der «Anderen», die häufig vorkamen (z.B. Natur vs. Kultur, aktiv vs. passiv, gesund vs. krank, Helfer vs. Opfer). Nach solchen gegensätzlichen Konstruktionen wollte ich in der Analyse Ausschau halten. Als Basis definierte ich drei Grundfragen:

- Welches Bild der «Anderen» wird vermittelt?

-

5 Carolin Philipp, «Teilen sagt nicht über Anteile aus». Die Konstruktion von Weisssein und Schwarzsein auf Plakaten von «Hilfsorganisationen», in: Interkultureller Rat in Deutschland (Hg.), Materialheft zu den Internationalen Wochen gegen Rassismus $2012,20-23$. 
- In welchem Verhältnis steht dieses Bild der «Anderen» zum implizierten Selbstbild?

- Werden globale Zusammenhänge thematisiert?

Je mehr dabei bei der Analyse die inhaltlichen Gegensätze nach Philipp auftauchen und je stärker diese ausgeprägt sind, desto weniger postkolonial sensibel scheint die Kampagne.

Sowohl Sprache als auch das Bildmaterial der Kampagnen sollten in der Analyse der HEKS-Kampagnen Beachtung finden.

Im Folgenden werden die wichtigsten Erkenntnisse aus der Analyse kurz zusammengefasst.

\section{Von bilfsbedürttigen Bauern und mutigen Aktivistinnen}

Mit Blick auf die Konstruktion der «Anderen» ergaben sich in der Analyse der drei Kampagnen grosse Unterschiede. In der Kampagne «Hilfe schenken» konnten mehrere der in der Methodik festgehaltenen Gegensätze in z.T. deutlicher Ausprägung erkannt werden. So werden z.B. durch die Art der Geschenke, die beschreibenden Texte und nicht zuletzt durch Struktur und Titel der Kampagne die «Anderen» vor allem als hilflose Opfer konstruiert. Ein gutes Beispiel zur Veranschaulichung ist das «Plumpsklo» (Abb. 2) $)^{6}$ :

«Der umgangssprachlich geprägte Begriff 〈Plumpsklo» weckt deutlich unangenehmere Assoziationen als das im Text [Innenseite der Karte] verwendete Wort Latrine. De Titel zum Text lautet: Säubern Sie die Welt von Krankheiten.» Diese direkte Handlungszuschreibung verstärkt wiederum die als aktiv konstruierte Rolle der Betrachtenden. Das Bild zeigt eine kleine Hütte mit Strohdach, die für uns an Toiletten Gewöhnte nicht gerade einladend aussieht. Überspitzt ausgedrückt könnte die Botschaft auch so verstanden werden: \ank mir können diese armen Menschen zumindest auf ein

6 Hilfswerk der Evangelischen Kirchen Schweiz (HEKS), Hilfe schenken, Plumsklo. Befreien Sie die Welt von Krankheiten, URL: https://www.hilfe-schenken.ch/geschenke/detail/plumpsklo/ (abgerufen am 13.03.2021).
Plumpsklo gehen und müssen ihre Notdurft nicht irgendwo erledigen. Eine Botschaft, die sehr klare Aktiv / Passiv- und Helfer / Opfer-Verhältnisse schafft. Auch die Dimension von gesund und krank spielt hier hinein. Damit verbunden ist im weiteren Sinne auch das (S̈ubern: Uns als Betrachtende wird das Säubern zugeschrieben, was uns selber mit Reinheit verbindet. Die Krankheiten hingegen und damit das Schmutzig-Sein wird mit den Empfangenden assoziiert., ${ }^{7}$

Damit lässt sich mit Blick auf die gewählte Methodik diese Kampagne als am wenigsten postkolonial sensibel werten.
Jahrbuch Diakonie Schweiz 4 (2020/2021) - ISSN 2504-3994

$$
\text { CC by } 4.0
$$

Jahrbuch Diakonie Schweiz 4 (2020/2021) http://dx.doi.org/10.22018/JDS.2021.15 


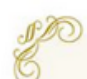

ese

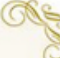

\section{SCHENKUngSuRKunde}

FÜR EIN

\section{Plumpsklo}

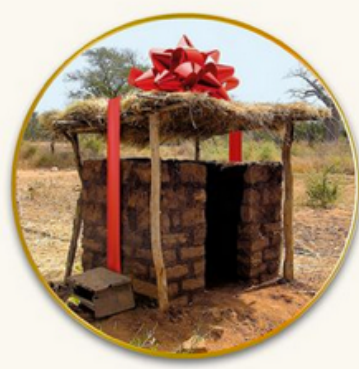

www.hilfe-schenken.ch
Die Indien-Kampagne erscheint in der Konstruktion der «Anderen» postkolonial sensibler - hier werden auch einheimische Fachpersonen gezeigt und die Adivasi werden ebenfalls als aktiv dargestellt. Als postkolonial vorbildhaft kann die Kolumbien-Kampagne gelten. In ihr lässt sich keiner der methodisch definierten Gegensätze erkennen. Es stehen ganz und gar die dortigen Aktivistinnen im Fokus, sie werden als starke Frauen porträtiert, über deren Mut wir nur staunen können.

Von grosszügiger Hilfe zu relativierter Unterstützung

Auch beim implizierten Selbstbild bleiben die Unterschiede gross.

In der ersten Kampagne besteht eine klare Rollen-Hierarchie zwischen mir als betrachtender Person und den implizierten (Anderens, Das (Wir) wird als aktiv helfend und grosszügig schenkend konstruiert. Ganz anders in der Kampagne über Kolumbien: Den Betrachtenden wird klar die Rolle der Unterstützenden zugewiesen und die eigenen Möglichkeiten werden relativiert. Diejenigen, die wirklich aktiv sind und sich einsetzen, sind die Frauen in den Projekten. Bei der dritten Kampagne könnte von einer leichten Unausgeglichenheit der Rollen gesprochen werden, da ein Stück weit ein Geber / Nehmer-Verhältnis zur Wirkung kommt; im Gegensatz zur ersten Kampagne stehen aber trotzdem die Leute in den Projekten im Fokus und nicht ich als Betrachterin. ${ }^{8}$

Globale Verflechtungen?

Dieser Punkt lässt sich einheitlich beantworten: Keine der drei Kampagnen greift globale Verflechtungen auf. Die einzige Verbindung von hier in

8 Knobel, Von hilflosen Bauern (Anm. 1), 83 
die Projektländer, die geschaffen wird, ist diejenige der Hilfe und Unterstützung - damit wird das Machtgefälle verstärkt.

\section{Marketing vs. Sensibilität?}

Ich möchte hier festhalten, was mir auch in der Arbeit wichtig zu betonen war: Die Kritik stellt keinen Vorwurf dar, auch die guten Absichten hinter den Kampagnen oder eine postkoloniale Sensibilität innerhalb der Projektarbeit wird nicht bestritten. Das Dekolonisieren von Gedankenmustern und Bildern ist vielmehr ein langwieriger Prozess, zu dem ich ermutigen möchte. Dabei ist mir klar, dass gute Marketing-Gründe gegen meine Einwände vorgebracht werden könnten: So ist z.B. eine stark hervorgehobene Dankbarkeit der Unterstützten sicherlich werbewirksam, und weshalb sollte der Titel einer gut laufenden Kampagne wie «Hilfe schenken» geändert werden? Ich vertrete dazu folgende Ansicht:

Es ist jedoch meine persönliche Überzeugung, dass zu Gunsten des Marketings keine Kompromisse gemacht werden dürfen, die zu Lasten der Menschen in den Projekten und unserer Wahrnehmung von ihnen gehen. Vielleicht können intensive Bemühungen eines Hilfswerks, wirklich so weit wie irgend möglich von den Menschen in den eigenen Projekten auszugehen und eine postkoloniale Sensibilität durchzusetzen, im Gegenzug irgendwann auch einen Marktvorteil bringen. Die Analyse zeigt zudem, das gerade bei Teilen der Kampagne, die durch kürzere Informationen wirken müssen (z.B. Plakat), die Gefahr stereotyper Bilder besonders gross ist. Hier braucht es vermutlich auch Kreativität, um Lösungen zu finden. Das Abwechseln von Kampagnen mit unterschiedlichen Themen kann ebenfalls zu weniger stereotypen Bildern beitragen (wie eben z.B. die Indien-Kampagne, die als Nachfolgekampagne auf Kolumbien folgte). Auch das Variieren von Bildern innerhalb einer Kampagne könnte hilfreich sein.

Es bleibt jedoch besonders festzuhalten, dass sehr vieles v.a. in der Indien- und in der Kolumbien-Kampagne bereits aus postkolonialer Sicht sehr sensibel gestaltet ist. Auch kamen die meisten der von Philipp genannten Problemfelder kaum oder nur am Rande zum Vorschein. Gerade «Gloria - Frauen für den Frieden in Kolumbien` zeigt, wie aus postkolonialer Sicht eine sehr gute Kampagne gestaltet werden kann; ihr würde ich deshalb sogar eine Vorbildfunktion zuschreiben. Der Prozess des kulturellen Dekolonisierens, eben z.B. durch die in Kampagnen vermittelten Bilder der (Anderens, ist ein anhaltender und kein einfacher Vorgang. Es gilt $m$.E. aber kontinuierlich dran zu bleiben. ${ }^{9}$

\section{Fazit und Ausblick}

Ich fasse einige wichtige Punkte zusammen, die sich in der Arbeit in Bezug auf die Leitfrage ergeben haben:

- Die Struktur der Ökumene selber fördert eine postkoloniale Empfindsamkeit. Die verschiedenen Kirchen des Nordens und Südens sind in ihr gleichberechtigte Partnerinnen. Das theologische Motiv des einen Leibes Christi ist in diesem Zusammenhang ein interessantes Bild, das dies verdeutlichen könnte.

- Es hat sich zudem in der Arbeit gezeigt, dass postkoloniale Theorien ein praxisfähiges, kritisches Analyseinstrumentarium bilden können.

- In der Fusion von HEKS und Bfa vermute ich eine postkoloniale Chance, da durch diese entwicklungspolitische Sensibilität und Projektarbeit stärker zusammenrücken.

- Als Grenze postkolonialer Theorien gilt festzuhalten, dass sie kritisieren, ohne selbst ein positives Narrativ zu bieten. Diese positiven Narrative, die postkolonial anschlussfähig sind, können aber an anderen Orten gefunden werden. Eines, welches bereits stark in der christlichen Tradition verankert ist, wäre z.B. die Gleichheit von unten. Das zeigt: Vielleicht müssen gar keine neuen Narrative erfunden werden, sondern passende neu betont.

9 Knobel, Von hilflosen Bauern (Anm. 1), 84.
Jahrbuch Diakonie Schweiz 4 (2020/2021) - ISSN 2504-3994

$$
\text { CC by } 4.0
$$

Jahrbuch Diakonie Schweiz 4 (2020/2021) http://dx.doi.org/10.22018/JDS.2021.15 
- Ich möchte mich für den Mut, sich auch in ökonomisch schwierigen Zeiten zu dekolonisieren, stark machen. Dafür braucht es

Kreativität und eine andauernde Suche nach guten Lösungen.

Zum Ausblick:

- Die postkoloniale Betrachtungsmöglichkeit kirchlicher EZA ist mit der Arbeit noch lange nicht ausgeschöpft. Alle Arbeitsbereiche, z.B. auch die Projektarbeit, oder die Struktur der Organisation könnten so untersucht werden - es handelt sich um ein umfangreiches Unterfangen. In einer geplanten Dissertation möchte ich dies noch umfassender analysieren.

- Auch grundsätzlich lohnt es sich weiterhin, postkoloniale Theorien für die Theologie stark zu machen. Denn Theologie ist m.E immer noch häufig eurozentrisch geprägt.

Zu guter Letzt ist ein Grundwunsch, der hinter der diesem Beitrag zugrunde liegenden Masterarbeit liegt und darüber hinausführt, mehr strukturelle Gleichberechtigung, wie sie eben auch von der «Black Lives Matter»-Bewegung gefordert wird. Der mit Abstand grösste Teil des heutigen Rassismus findet sich in unterbewussten Stereotypen und diese beeinflussen unsere Systeme. Schwindende stereotype Denkmuster bedeuten demnach mehr strukturelle Gleichberechtigung - und diese sollte ein wichtiges Ziel institutioneller Diakonie sein. Für Diakonie im Bereich der EZA ist demnach eine postkoloniale Sensibilität unumgänglich, wenn sie zeitgemäss und engagiert bleiben will.

Autorin:

Isabelle Knobel studierte Theologie an der Universität Bern und ist Vikarin in der Kirchgemeinde Heiliggeist, Bern 